Durch Vergleich der LDH-Aktivität bei verschiedenen Konzentrationen an Pyruvat $(29,30)$ oder Lactat (31) ist somit der Rückschluß möglich, ob eine Erhöhung der LDH-Aktivität hepatogener oder kardialer Genese ist.

Diese Tatsache wird für die Bestimmung der , $\alpha$ Hydroxybutyratdehydrogenase" mit $\alpha$-Ketobutyrat als Substrat angewendet.

Durch eine niedrige Substratkonzentration wird eine bevorzugte Umsetzung des $\alpha$-Ketobutyrats durch die für Herzerkrankungen typische $\mathrm{LDH}_{1}$ erreicht. Je nach ihren Anteilen an der Gesamtaktivität sind aber auch die anderen Isoenzyme an der „HBDH-Aktivität" beteiligt
(23). Die Bestimmung bei nichtoptimaler Substratkonzentration ergibt nichtoptimale Aktivitätswerte.

Bei optimaler Substratkonzentration ist nach unseren Untersuchungen die mit Pyruvat und $\alpha$-Ketobutyrat gemessene LDH-Aktivität gleich groß. Unsere Versuche mit Inhibitoren bestätigen ebenfalls die völlige Übereinstimmung.

Eine Bestimmung der LDH-Aktivität mit $\alpha$-Ketobutyrat sollte daher auch nicht mehr als $\alpha$-Hydroxybutyratdehydrogenase-Aktivität bezeichnet werden, da sich für die Existenz einer „ $\alpha$-Hydroxybutyratdehydrogenase" im Serum kein Anhalt ergibt.

\title{
Literatur
}

1. Wilkinson, J.H. und W. A. Withycombe, Biochem. J. 97, 663 (1965). - 2. FreemanN, J. und A. W. Opler, Amer. J. med. Sci. 250, 131 (1965). - 3. Rosalki, S. B., J. Clin. Path. 15, 566 (1962). -4. Rosalki, S. B. und J. H. Wilkinson, Nature (London) 188, 1110 (1960). - 5. Dubach, U. C. und L. Margreth, Dtsch. med. Wschr. 90, 1429 (1965). - 6. Stuart, J., J. C. Grawford, J. Forshall und I. A. Owen, Brit. Med. J. I 423 (1965). - 7. Forster, G. und S. FeissLr, Helvet. med. Acta 31, 389 (1964). 8. Schneider, K. W., F.-G. Lehmann und G. Hornung, Arch. Kreislaufforsch. 46, 306 (1965). - 9. WroBlewsKr, F. und J. S. LA Due, Proc. Soc. exp. Biol. Med. 90, 210 (1965). - 10. Bergmayer, H.-U., Methoden der enzymatischen Analyse, Verlag Chemie GmbH., Weinheim/Bergstr. (1962). - 11. PAwCETr, C. P. und M. M. Crotrr, ,Biochim. biophysica Acta (Amsterdam) 54, 210 (1961). - 12. Richterich, R., P. Schafroth und H. Aebi, Clin. chim. Acta (Amsterdam) 8, 178 (1963). - 13. HsieH, K. M. und H. T. Blumenthal, Proc. Soc. exp. Biol. Med. 91, 626 (1956). 14. Wieland, T., G. PfLeiderer und F. Ortandert, Biochem. $Z$. 331, 103 (1959). - 15. Eherson, P. M. und J. H. Wilkinson, J. Clin. Path. 18, 803 (1965). - 16. Warburton, F. G. und D.
SMrth, Enzymologia 26, 125 (1963/64). - 17. Plumare, D. T. J. H. Wilkinson und W. A. Withycombe, Biochem. J. 89, 48P (1963). - 18. KontrineN, A. und S. LiNDY, Nature (London) 208, 782 (1965). - 19. HARDY, S. M., Nature (London) 206, 933 (1965). - 20. Reitmann, S. und S. Frankel, Amer. J. Clin. Path. 28, 56 (1957). - 21. Elliotr, B. A. und J. M. Wirkinson, Lancet (London) I, 698, 1961. - 22. Stambough, R. und D. Post, J. biol. Chemistry 241, 1462 (1966). - 23. Rosalkr, S. B., Clin. chim. Acta (Amsterdam) 8, 415 (1963). - 24. Plummer, D. T., B. A. Elliott, K. B. Cooke und J. H. Wilkinson, Biochem. J. 87, 416 (1963). - 25. WithyсомвE, W. A. und J. H. Wilkinson, Biochem. J. 89, 48P (1963). - 26. Hule, V., Clin. chim. Acta (Amsterdam) 13, 431 (1966). - 27. Czok, R. und T. Bücher, Advances Protein Chem. 15, 365 (1960). - 28. Nisselbaum, J. S., D. E. PACKER und O. Bodansky, J. biol. Chemistry 239, 2830 (1964). 29. Plagemann, P. G. W., K. F. Gregory und F. Wroblewski, J. biol. Chemistry 235, 2288 (1960). - 30. CaHN, R. D., N. O. Kaplan, L. Levine und E. Zwilling, Science (Washington) 136, 962 (1962). - 31. Babson, A. L. und G. E. Phillirs, Clin. chim. Acta (Amsterdam) 12, 210 (1965).

\section{Experimentelle Untersuchungen zum Verfahren der in-vitro-Lipolyse}

\author{
Von P. Schwandt, M. KNedel und R. Lindlbauer
}

Aus der I. Medizinischen Klinik der Universität München (Direktor: Prof. Dr. H. Schwiegk) und dem Klinisch-Chemischen Institut des Städtischen Krankenbauses München-Harlacbing (Chefarzt: Priv.-Doz. Dr. M. Knedel)

\author{
(Eingegangen am 24. August 1967)
}

Am epididymalen Fettgewebe der Ratte wurde die Wirkung verschiedener Inkubationsbedingungen auf die spontane und hormoninduzierte in-vitro-Lipolyse untersucht. Nach genauer Beschreibung des Versuchsansatzes werden die Einflüsse von Temperatur, Fettgewebsmenge, Inkubationszeit, Albuminkonzentration und Pufferzusammensetzung mitgeteilt. Daraus ergeben sich optimale Versuchsbedingungen für die in-vitro-Lipolyse, deren Standardisierung als notwendig erachtet wird.

Investigations are reported on the influence of different incubation conditions on the spontaneous and hormone-induced lipolysis in epididymal fat pads of the rat. The incubation technique is described in detail, and the effects of temperature, quantity of adipose tissue, incubation time, concentration of albumin and the ionic composition of the buffer are reported. The optimal conditions were thus established and their standardisation is recommended for the in vitro study of lipolysis.

Das Fettgewebe ist metabolisch hoch aktiv, relativ uniform und leicht zugänglich. Es bietet sich daher für die Durchführung von Stoffwechseluntersuchungen als geeignetes Material an. Wegen seiner Struktur trifft das vor allem auf das epididymale Fettgewebe der Ratte $z u$, das besonders häufig für die Untersuchung lipolytischer Vorgänge in vitro herangezogen wurde. Die ursprünglich von Gordon und Cherkes (1) und White und 
ENGEL (2) angegebene Inkubationstechnik unter Úberlebensbedingungen wurde in mehrfach modifizierter Form angewendet.

$\mathrm{Da}$ zusammenfassende Untersuchungen über die Wirkung einzelner Bedingungen des Inkubationsvorganges auf die Lipolyse fehlen, hat diese Arbeit zum Ziel, den Einfluß von Temperatur, Inkubationsdauer, Albuminkonzentration, Fettgewebsmenge und Ionenkonzentration $\mathrm{zu}$ untersuchen.

\section{Methode und Material}

Entsprechend dem früher beschriebenen Vorgehen (3) verwandten wir für unsere Untersuchungen die distal der Gefäße gelegenen Anteile des epididymalen Fettgewebes von etwa $500 \mathrm{~g}$ schweren Wistar-Ratten, die mit Altromin-Pellets (Fa. Altromin, Lage) ad libitum gefüttert waren.

Das Gewebe wurde gleich nach dem Töten der Tiere entnommen, in physiologischer Kochsalzlösung gespült und nach kurzem Trocknen auf Filterpapier in Portionen zu etwa $250 \mathrm{mg}$ abgewogen. Die Inkubation erfolgte in Warburg-Gefäßen, die je $3 \mathrm{ml}$ eines albuminhaltigen Krebs-Ringer-Bicarbonat-Puffers von pH 7,4 enthielten; dieser hatte folgende Zusammensetzung (in $\mathrm{mMol} / \mathrm{l}$ ): $106 \mathrm{NaCl}, 40 \mathrm{NaHCO}_{3}, 5,4 \mathrm{KCl}, 1,0 \mathrm{CaCl}_{2}, 1,0 \mathrm{KH}_{2} \mathrm{PO}_{4}$ und $1,0 \mathrm{MgSO}_{4}$. Das Rinderalbumin, das - wenn nicht anders angegeben - in einer Konzentration von $5 \mathrm{~g} / 100 \mathrm{~m} / \mathrm{im}$ Puffer gelöst war, wurde zuvor nach CAMpBELL (4) fettsäurefrei gewaschen: $10 \mathrm{~g}$ trockenes Albumin (Rinderalbumin trocken-reinst, Behringwerke, Marburg) wurden $15 \mathrm{Min}$. bei $0^{\circ} \mathrm{mit} 100 \mathrm{ml}$ 95proz. Äthanol-Äther $(3: 1 / v: r)$ gerührt, filtriert, mit wasserfreiem Äther mehrfach nachgewaschen und im Vakuum getrocknet.

Vor Beginn der Inkubation, die im allgemeinen 3 Stdn. bei $37^{\circ}$ und bei einer Schüttelfrequenz von $60 \mathrm{Min}$. und einer Amplitude von $2 \mathrm{~cm}$ in der Warburg-Apparatur durchgeführt wurde, wurde durch die Gefäße ein Gasgemisch aus $95 \% \mathrm{O}_{2}$ und $5 \% \mathrm{CO}_{2}$ geleitet; die Gefäße wurden anschließend luftdicht verschlossen. $\mathrm{Zu}$ vor waren dem Inkubationsmedium die jeweils angegebenen Mengen an lipolytischem Hormon und das gewogene Fettgewebe zugegeben worden.

Die bei der durch Hormone stimulierten Lipolyse jeweils zugesetzten maximal und minimal wirksamen Dosen entsprachen den Angaben von Rudman (5): Adrenalin (Suprarenin, Hoechst) min. $0,5 \mu \mathrm{g} / 3 \mathrm{~m} l$, max. $15 \mu \mathrm{g} / 3 \mathrm{ml} ;$ ACTH (ACTH, Schering) min. $0,5 \mu \mathrm{g} / 3 \mathrm{ml}, \max .45 \mu \mathrm{g} / 3 \mathrm{ml}$.

Am Ende der Inkubationszeit wurde das Fettgewebe möglichst rasch aus den Gefäßen entfernt und im Medium die Bestimmung der freien Fettsäuren nach DoLE (6) mit Heptan-gewaschenem Nilblau A als Indikator durchgeführt. Die Berechnung erfolgte in $\mathrm{mVal}$ freie Fettsäuren/g Fettgewebe/Std.

Die Standardabweichung wurde berechnet als $s=\sqrt{\frac{\sum(\mathrm{x}-\overline{\mathrm{x}})^{2}}{\mathrm{n}-1}}$. Die Wahrscheinlichkeit $\mathrm{p}$ wurde nach dem $\mathrm{t}$-Test ermittelt.

Den Einfluß der Inkubationszeit auf die spontane und hormoninduzierte Lipolyse untersuchten wir über einen Zeitraum von $5 \mathrm{Stdn}$., wobei in 1stdg. Abständen die freien Fettsäuren im Medium bestimmt wurden.

Die Bedeutung der Albuminkonzentration für die mit $10 \mu \mathrm{g} / 3 \mathrm{~m} l$ Adrenalin stimulierte Lipolyse prüften wir mit $2 \mathrm{~g} / 100 \mathrm{~m} l \mathrm{bzw}$. $5 \mathrm{~g} / 100 \mathrm{ml}$ Albuminzusatz zum Puffer.

Der Temperatureinflu $\beta$ auf die spontane und mit $5 \mu \mathrm{g} / 3 \mathrm{~m} /$ Adrenalin stimulierte Lipolyse wurde bei folgenden Temperaturen untersucht: $5^{\circ}, 23^{\circ}, 30^{\circ}, 32^{\circ}, 34^{\circ}, 36^{\circ}, 38^{\circ}, 40^{\circ}, 42^{\circ}$ und $44^{\circ}$. Geprüft wurde die Beziehung zwischen Fettgevebsmenge und Freisetzung freier Fettsäuren ohne Hormonzusatz und mit $10 \mu \mathrm{g} / 3 \mathrm{ml}$ Adrenalin. Die jeweils inkubierten Fettgewebsmengen betrugen 50, 100, 150,200 und $250 \mathrm{mg}$.

Der Einfluß verschiedener Kationenkonzentrationen im Inkubationsmedium auf die hormoninduzierte Lipolyse wurde durch Variation der Konzentrationen von Calcium, Kalium, Natrium und $\mathrm{Ma-}$ gnesium im Puffer untersucht: dem ohne Calcium angesetzten Puffer wurde in jeweils um $1 \mathrm{mVal} / \mathrm{l}$ steigender Konzentration von $0-10 \mathrm{mVal} / /$ Calcium zugesetzt, die Natriumkonzentration wurde in dem mit $120 \mathrm{mVal} / /$ angesetzten Puffer durch Zugabe von jeweils zusätzlich $5 \mathrm{mVal} / l$ pro Ansatz bis auf $170 \mathrm{mVal} / \mathrm{l}$ erhöht,

die Kaliunkonzentration betrug zwischen 0,4 und $10,4 \mathrm{mVal} / l$, wobei sich die einzelnen Konzentrationen pro Ansatz um jeweils $1 \mathrm{mVal} / \mathrm{l}$ unterschieden,

bei Magnesium betrugen die einzelnen Konzentrationsunterschiede jeweils $0,3 \mathrm{mVal} / l \mathrm{im}$ Bereich zwischen $0 \mathrm{mVal} / l$ und $3 \mathrm{mVal} / l$.

An Hormonen wurden $10 \mu \mathrm{g} / 3 \mathrm{~m} l$ Adrenalin bzw. $10 \mu \mathrm{g} / 3 \mathrm{~m} l$ ACTH zugegeben.

\section{Ergebnisse}

Tabelle 1 gibt die Mitțelwerte von jeweils 5 spontanen und hormoninduzierten Lipolysen in ihrem zeitlichen Verlauf wieder. Bei der Spontanlipolyse und der mit den minimal wirksamen Dosen von ACTH und Adrenalin bewirkten Lipolyse ist ein kontinuierlicher Anstieg der freien Fettsäuren über den gesamten Zeitraum von 5 Stdn. zu beobachten. Bei den maximal wirksamen Dosen der beiden zugesetzten Hormone dagegen ist in den ersten 3 Stdn. ein wesentlich steilerer Anstieg der freien Fettsäuren festzustellen; in den folgenden 2 Stdn. ist die Freisetzung der freien Fettsäuren wesentlich geringer.

Tab. 1

Freisetzung freier Fettsäuren während 5 Stdn. bei spontaner und bei der mit maximal bzw. minimal wirksamer Dosis von Adrenalin bzw. ACTH stimulierten Lipolyse. Jeder Wert ist das Mittel aus 5 Versuchen. Freie Fettsäuren in $\mathrm{mVal} / \mathrm{g} / \mathrm{Std}$.

\begin{tabular}{|c|c|c|c|c|c|}
\hline $\begin{array}{c}\text { Inkubationsdauer } \\
\text { (Stdn.) }\end{array}$ & 1 & 2 & 3 & 4 & 5 \\
\hline Spontanlipolyse & $\begin{array}{r}0,41 \\
\pm 0,04\end{array}$ & $\begin{array}{r}1,01 \\
\pm 0,12\end{array}$ & $\begin{array}{r}1,52 \\
\pm 0,20\end{array}$ & $\begin{array}{r}1,98 \\
\pm 0,19\end{array}$ & $\begin{array}{r}2,56 \\
\pm 0,20\end{array}$ \\
\hline $\begin{array}{l}\text { Adrenalin } \\
\text { maximal wirk- } \\
\text { same Dosis } \\
\text { minimal wirk- } \\
\text { same Dosis }\end{array}$ & $\begin{array}{r}2,05 \\
\pm 0,33 \\
0,92 \\
\pm 0,19\end{array}$ & $\begin{array}{r}5,06 \\
\pm 0,86 \\
2,38 \\
\pm 0,38\end{array}$ & $\begin{array}{r}9,31 \\
\pm 0,46 \\
3,13 \\
\pm 0,29\end{array}$ & $\begin{array}{r}9,26 \\
\pm 1,40 \\
5,14 \\
\pm 0,85\end{array}$ & $\begin{array}{r}9,95 \\
\pm 0,60 \\
6,87 \\
\pm 0,62\end{array}$ \\
\hline $\begin{array}{c}\text { ACTH } \\
\text { maximal wirk- } \\
\text { same Dosis } \\
\text { minimal wirk- } \\
\text { same Dosis }\end{array}$ & $\begin{array}{r}2,94 \\
\pm 0,82 \\
1,01 \\
\pm 0,26\end{array}$ & $\begin{array}{r}5,56 \\
\pm 0,67 \\
1,50 \\
\pm 0,07\end{array}$ & $\begin{array}{r}8,27 \\
\pm 0,74 \\
2,51 \\
\pm 0,19\end{array}$ & $\begin{array}{r}9,18 \\
\pm 0,77 \\
4,03 \\
\pm 0,47\end{array}$ & $\begin{array}{r}9,58 \\
\pm 0,93 \\
4,80 \\
\pm 0,49\end{array}$ \\
\hline
\end{tabular}

Der Einfluß der unterschiedlichen Albuminkonzentration im Inkubationsmedium geht aus Abbildung 1 hervor: während der 3stdg. Inkubationsdauer stiegen bei 5proz. Albuminkonzentration die freien Fettsäuren linear zur Zeit an; bei 2proz. Albuminzusatz war das Maximum der Freisetzung an freien Fettsäuren schon nàch 2 Stdn. erreicht, der weitere Anstieg um 0,16 mVal $/ l$ war nicht mehr signifikant.

Bei der Untersuchung des Temperatureinflusses auf die Freisetzung freier Fettsạuren konnte sowohl für die spontane als auch für die durch Adrenalin induzierte Lipolyse im Bereich von $30^{\circ}-44^{\circ}$ kein systematischer ${ }^{\circ}$ Einfluß festgestellt werden (Tab. 2). Bei $23^{\circ}$ ist die Freisetzung der freien Fettsäuren vermindert, wobei die hormoninduzierte Lipolyse mit $0,63 \mathrm{mVal} / l$ deutlich höher lag als die ohne Hormonźugabe. Die Spontanlipolyse entsprach mit $0,22 \mathrm{mVal} / l$ bei $23^{\circ}$ dem bei $25^{\circ}$ gefundenen Wert.

Bis zu $150 \mathrm{mg}$ Fettgewebe war das Ausmaß der Freisetzung freier Fettsäuren eindeutig von der zugegebenen 


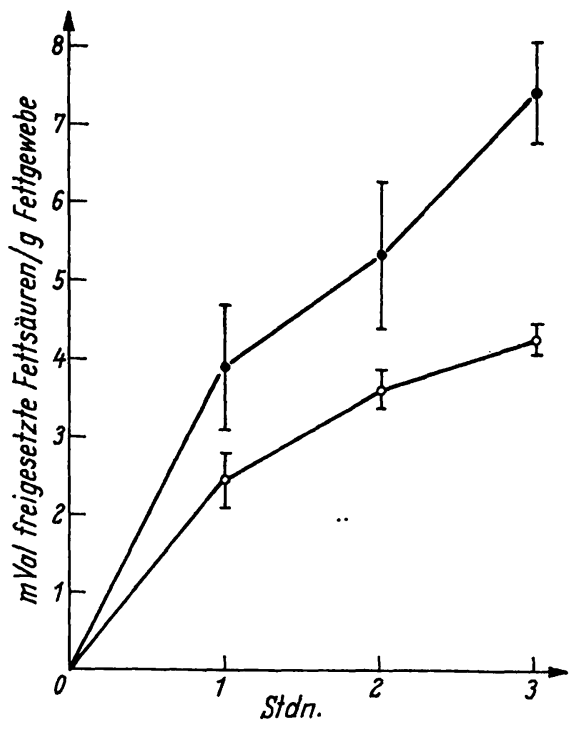

Abb. 1

Zusammenhang zwischen Albuminkonzentration im Medium und Freisetzung freier Fettsäuren. Jeder Punkt stellt den Mittelwert aus 5 Untersuchungen, jede senkrechte Linie die Standardabweichung dar.

- Albuminkonzentration 5\%, ๑- Albuminkonzentration $2 \%$

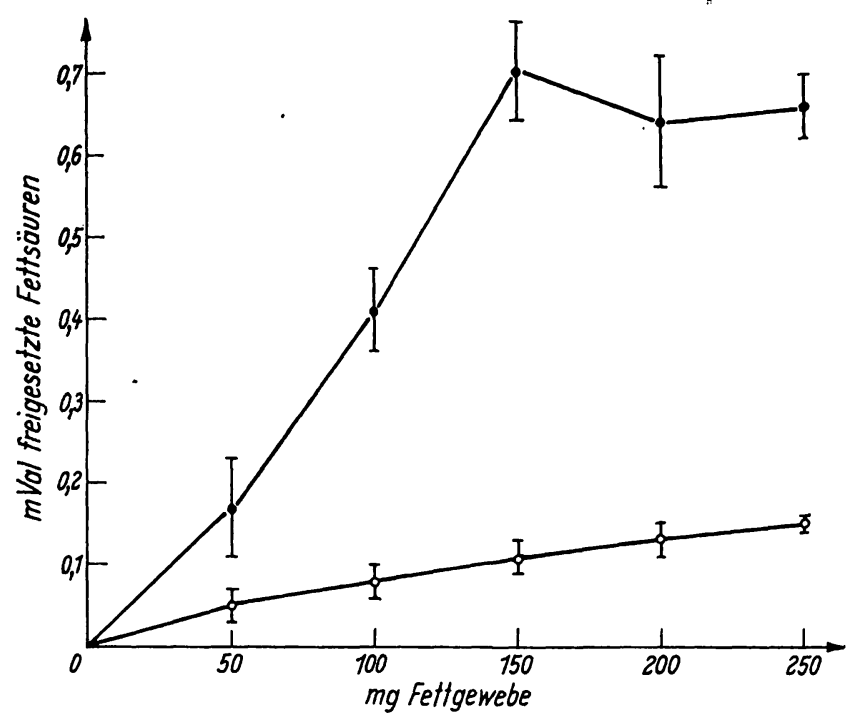

Abb. 2

Relation zwischen Freisetzung freier Fettsäuren und Fettgewebsmenge. Bei der mit $10 \mu \mathrm{g}$ Adrenalin stimulierten Lipolyse (•-•) stellt jeder Punkt den Mittelwert aus 7, bei der Spontanlipolyse $(\circ-0)$ aus 6 Versuchen, die senkrechte Linie die Standardabwreichung dar

Tab. 2

Einfluß der Temperatur auf die spontane und die adrenalininduzierte Lipolyse. Bei der Spontanlipolyse stellt jeder Wert das Mittel aus 4 , bei der Adrenalinlipolyse aus 7 Versuchen dar. Freie Fettsäuren in $\mathrm{mVal} / \mathrm{g} / \mathrm{Std}$

\begin{tabular}{|c|c|c|c|c|c|c|c|c|c|c|}
\hline Temperatur & $5^{\circ}$ & $23^{\circ}$. & $30^{\circ}$ & $32^{\circ}$ & $34^{\circ}$ & $36^{\circ}$ & $38^{\circ}$ & $40^{\circ}$ & $42^{\circ}$ & $44^{\circ}$ \\
\hline Spontanlipolyse & $\begin{array}{r}0,23 \\
\pm 0,01\end{array}$ & $\begin{array}{r}0,22 \\
\pm 0,01\end{array}$ & $\begin{array}{r}0,33 \\
\pm 0,03\end{array}$ & $\begin{array}{r}0,55 \\
\pm 0,05\end{array}$ & $\begin{array}{r}0,34 \\
+0,04\end{array}$ & $\begin{array}{r}0,54 \\
+0,04\end{array}$ & $\begin{array}{r}0,49 \\
\pm 0,07\end{array}$ & $\begin{array}{r}0,85 \\
\pm 0,02\end{array}$ & $\begin{array}{r}0,49 \\
\pm 0,06\end{array}$ & $\begin{array}{r}0,67 \\
\pm 0,05\end{array}$ \\
\hline Adrenalinlipolyse & $\begin{array}{r}0,31 \\
\pm 0,05\end{array}$ & $\begin{array}{r}0,63 \\
\pm 0,16\end{array}$ & $\begin{array}{r}1,25 \\
\pm 0,14\end{array}$ & $\begin{array}{r}2,00 \\
\pm 0,30\end{array}$ & $\begin{array}{r}1,26 \\
\pm 0,07\end{array}$ & $\begin{array}{r}1,34 \\
\pm 0,24\end{array}$ & $\begin{array}{r}1,11 \\
\pm 0,25\end{array}$ & $\begin{array}{r}1,28 \\
\pm 0,21\end{array}$ & $\begin{array}{r}1,17 \\
+0,13\end{array}$ & $\begin{array}{r}1,12 \\
\pm 0,05\end{array}$ \\
\hline
\end{tabular}

Tab. 3

Einfluß verschiedener Kationenkonzentrationen im Inkubationsmedium auf die mit Adrenalin bzw. ACTH stimulierte Lipolyse. Jeder Wert ist das Mittel aus 3, bei Natrium aus 5 Versuchen. Freie Fettsäuren in $\mathrm{mVal} / \mathrm{g} / \mathrm{Std}$.

\begin{tabular}{lccccccccccc}
\hline Natrium (mVal/l) & 120 & 125 & 130 & 135 & 140 & 145 & 150 & 155 & 160 & 165 & 170 \\
\hline Adrenalin & 2,71 & 3,33 & 3,38 & 3,51 & 3,40 & 2,92 & 3,29 & 3,30 & 2,42 & 2,81 & 2,91 \\
& $\pm 0,29$ & $\pm 0,20$ & $\pm 0,74$ & $\pm 0,47$ & $\pm 0,71$ & $\pm 0,56$ & $\pm 0,78$ & $\pm 0,55$ & $\pm 0,51$ & $\pm 0,51$ & $\pm 0,68$ \\
ACTH & 3,59 & $\mathbf{3 , 4 2}$ & 3,35 & 1,04 & 3,68 & 2,52 & 2,69 & 2,63 & 3,08 & 2,77 & 2,53 \\
& $\pm 0,22$ & $\pm 0,23$ & $\pm 0,46$ & $\pm 0,64$ & $\pm 0,15$ & $\pm 0,56$ & $\pm 0,58$ & $\pm 0,55$ & $\pm 0,29$ & $\pm 0,52$ & $\pm 0,25$ \\
\hline \hline
\end{tabular}

\begin{tabular}{|c|c|c|c|c|c|c|c|c|c|c|c|}
\hline Calcium (mVal/l) & 0 & 1 & 2 & 3 & 4 & 5 & 6 & 7 & 8 & 9 & 10 \\
\hline Adrenalin & $\begin{array}{r}1,97 \\
\pm 0,34\end{array}$ & $\begin{array}{r}1,52 \\
\pm 0,27\end{array}$ & $\begin{array}{r}2,24 \\
\pm 0,51\end{array}$ & $\begin{array}{r}1,60 \\
\pm 0,07\end{array}$ & $\begin{array}{r}1,49 \\
\pm 0,31\end{array}$ & $\begin{array}{r}1,73 \\
\pm 0,28\end{array}$ & $\begin{array}{r}2,05 \\
\pm 0,48\end{array}$ & $\begin{array}{r}1,27 \\
\pm 0,22\end{array}$ & $\begin{array}{r}1,37 \\
\pm 0,22\end{array}$ & $\begin{array}{r}2,08 \\
\pm 0,87\end{array}$ & $\begin{array}{r}1,76 \\
\pm 0,53\end{array}$ \\
\hline $\mathrm{ACTH}$ & $\begin{array}{r}0,89 \\
\pm 0,20\end{array}$ & $\begin{array}{r}1,10 \\
\pm 0,31\end{array}$ & $\begin{array}{r}1,53 \\
\pm 0,31\end{array}$ & $\begin{array}{r}2,05 \\
\pm 0,31\end{array}$ & $\begin{array}{r}2,10 \\
\pm 0,31\end{array}$ & $\begin{array}{r}2,32 \\
\pm 0,25\end{array}$ & $\begin{array}{r}3,00 \\
\pm 0,24\end{array}$ & $\begin{array}{r}2,45 \\
\pm 0,10\end{array}$ & $\begin{array}{r}2,78 \\
\pm 0,60\end{array}$ & $\begin{array}{r}2,50 \\
\pm 0,59\end{array}$ & $\begin{array}{r}2,44 \\
\pm 0,76\end{array}$ \\
\hline Magnesium (mVal/l) & 0 & 0,3 & $0 ; 6$ & 0,9 & 1,2 & 1,5 & 1,8 & 2,1 & 2,4 & 2,7 & 3,0 \\
\hline Adrenalin & $\begin{array}{r}2,55 \\
\pm 0,32\end{array}$ & $\begin{array}{r}2,24 \\
\pm 0,35\end{array}$ & $\begin{array}{r}2,03 \\
\pm 0,44\end{array}$ & $\begin{array}{r}2,56 \\
\pm 0,73\end{array}$ & $\begin{array}{r}1,95 \\
\pm 0,61\end{array}$ & $\begin{array}{r}2,68 \\
\pm 0,27\end{array}$ & $\begin{array}{r}2,45 \\
\pm 0,57\end{array}$ & $\begin{array}{r}2,58 \\
\pm 0,16\end{array}$ & $\begin{array}{r}2,38 \\
+0,29\end{array}$ & $\begin{array}{r}2,88 \\
\pm 0,33\end{array}$ & $\begin{array}{r}2,61 \\
+0,38\end{array}$ \\
\hline ACTH & $\begin{array}{r}2,32 \\
\pm 0,23\end{array}$ & $\begin{array}{r}2,44 \\
\pm 0,13\end{array}$ & $\begin{array}{r}2,68 \\
\pm 0,11\end{array}$ & $\begin{array}{r}2,51 \\
+0,32\end{array}$ & $\begin{array}{r}1,94 \\
\pm 0,35\end{array}$ & $\begin{array}{r}2,25 \\
\pm 0,32\end{array}$ & $\begin{array}{r}2,43 \\
\pm 0,10\end{array}$ & $\begin{array}{r}2,28 \\
\pm 0,43\end{array}$ & $\begin{array}{r}2,35 \\
\pm 0,22\end{array}$ & $\begin{array}{r}2,54 \\
\pm 0,04\end{array}$ & $\begin{array}{r}2,29 \\
\pm 0,18\end{array}$ \\
\hline Kalium (mVal/l) & 0,4 & 1,4 & 2,4 & 3,4 & 4,4 & 5,4 & 6,4 & 7,4 & 8,4 & 9,4 & 10,4 \\
\hline $\begin{array}{l}\text { Adrenalin } \\
\text { ACTH }\end{array}$ & $\begin{array}{r}2,25 \\
\pm 0,44 \\
2,24 \\
\pm 0,24\end{array}$ & $\begin{array}{r}1,57 \\
\pm 0,09 \\
2,54 \\
\pm 0,51\end{array}$ & $\begin{array}{r}1,58 \\
\pm 0,32 \\
2,94 \\
\pm 0,23\end{array}$ & $\begin{array}{r}1,75 \\
\pm 0,27 \\
2,90 \\
\pm 0,22\end{array}$ & $\begin{array}{r}1,57 \\
\pm 0,15 \\
2,45 \\
\pm 0,55\end{array}$ & $\begin{array}{r}2,21 \\
\pm 0,58 \\
2,54 \\
\pm 0,40\end{array}$ & $\begin{array}{r}1,76 \\
\pm 0,52 \\
2,79 \\
\pm 0,17\end{array}$ & $\begin{array}{r}1,84 \\
\pm 0,54 \\
2,94 \\
\pm 0,23\end{array}$ & $\begin{array}{r}2,96 \\
\pm 0,80 \\
2,93 \\
\pm 0,35\end{array}$ & $\begin{array}{r}2,34 \\
\pm 0,51 \\
2,94 \\
\pm 0,09\end{array}$ & $\begin{array}{r}1,59 \\
\pm 0,13 \\
3,27 \\
\pm 0,23\end{array}$ \\
\hline
\end{tabular}

Substratmenge abhängig, wenn Adrenalin zugesetzt worden war. Größere Fettgewebsportionen führten zu keiner weiteren Steigerung. Anders dagegen verhielt sich die Spontanlipolyse, die auch bei $200 \mathrm{mg}$ einen weiteren Anstieg zeigte (Abb. 2).
Der Effekt verschiedener Ionenkonzentrationen im Inkubationsmedium auf die hormoninduzierte Freisetzung freier Fettsäuren geht aus Tabelle 3 hervor. Eine Beeinflussung durch Magnesium ergab sich in dem geprüften Bereich nicht. Kaliumkonzentrationen bis zu $10 \mathrm{mVal} / \mathrm{l}$ 
bewirkten dagegen eine zunehmende $L$ ipolyseste igerung von $2,24 \mathrm{mVal} / \mathrm{l}$ auf $3,27 \mathrm{mVal} / \mathrm{l}$ bei der durch $\mathrm{ACTH}$ induzierten Freisetzung freier Fettsäuren. Bei Adrenalinzusatz zeigte sich keine Beeinflussung der Lipolyse. Im Gegensatz zur adrenalininduzierten Lipolyse ist die durch ACTH hervorgerufene Freisetzung freier Fettsäuren auch durch Konzentrationsänderungen der anderen Ionenanteile $\mathrm{zu}$ beeinflussen. So hatte im Vergleich zu dem Bereich von 145-170 mVal/l eine niedrigere Natriumionenkonzentration (120-140 mVal/l) einen lipolysesteigernden Effekt. Am ausgeprägtesten war jedoch der Einfluß der Calciumionen, die bei einer Konzentration von $0-1 \mathrm{mVal} / \mathrm{lnur}$ eine minimale Steigerung der Freisetzung freier Fettsäuren bewirkten, während hohe Konzentrationen über den physiologischen Bereich hinaus $(6-10 \mathrm{mVal} / l)$ keinen weiteren steigernden Effekt hatten. Änderungen bei der adrenalininduzierten Lipolyse waren bei den verschiedenen Calciumkonzentrationen nicht $\mathrm{zu}$ beobachten.

\section{Diskussion}

Aus den vorliegenden Untersuchungen geht hervor, daß die Inkubationsbedingungen einen $z$. T. sehr wesentlichen Einfluß auf die in-vitro-Lipolyse im epididymalen Fettgewebe der Ratte haben. Das gilt sowohl für die Spontanlipolyse wie für die hormoninduzierte Freisetzung freier Fettsäuren. Zur Vermeidung von Fehlinterpretationen und zum besseren Vergleich der Ergebnisse verschiedener Autoren erscheint eine Standardisierung der Lipolysebedingungen unumgänglich.

Besonders in der Zusammensetzung der Puffer sind die Methoden noch keineswegs einheitlich: während die meisten Autoren mit Krebs-Ringer-Bicarbonat arbeiten, führen einige Autoren die in-vitro-Lipolysen mit KrebsRinger-Phosphat durch. Aus den Untersuchungen von LrNN (7) geht jedoch hervor, daß Phosphatpuffer weniger physiologisch ist, da er eine Hemmung der Triosephosphatdehydrogenase im Fettgewebe bewirkt. $\mathrm{Nach}$ Hirsch (8) ist die Anwesenheit von $\mathrm{HCO}_{3}$-Ionen für eine normale Lipidsynthese Voraussetzung. MosINGER (9) und LYNN (7) haben dagegen mit Phosphat-, Tris- und Bicarbonatpuffer keine unterschiedlichen Lipolyse-Ergebnisse beobachten können. Wesentlich für die Zusammensetzung des Puffers erscheint jedoch die Tatsache, daß er die gleiche Osmolarität wie das Serum hat, wobei die Eiweißkomponente durch Bicarbonat ersetzt wird (10).

$\mathrm{Daß}$ die Konzentration der einzelnen Ionenanteile im Puffer und nicht seine Osmolarität allein von entscheidender Bedeutung ist, geht aus den Befunden von LOPEz (11) hervor, der bei Fehlen von Calcium-Ionen mit ACTH keine Lipolyse erzielen konnte, wohl aber mit Adrenalin. Mit diesen Beobachtungen stimmen unsere Ergebnisse überein. Wie aus unseren Untersuchungen weiter hervorgeht, sind auch die Natriumund Kaliumkonzentrationen für die durch ACTH stimulierte Freisetzung freier Fettsäuren von Bedeutung. Bei niedrigen Natrium- und hohen Kaliumkonzentrationen fanden wir die Lipolyse um annähernd den gleichen
Wert gesteigert. Möglicherweise hängt das mit der Rolle zusammen, die diese Ionen als die wesentlichen intraund extrazellulären Kationen spielen, wobei eine hohe intrazelluläre Kaliumionen-Konzentration die Freisetzung freier Fettsäuren zu begünstigen scheint. Auch RoDberl (12) hat bei der Prüfung der Insulinempfindlichkeit des Fettgewebes festgestellt, daß Kalium und Natrium die wesentlichen Ionen des Inkubationsmediums darstellen. In Übereinstimmung mit MosInger (9) fanden wir bei der Änderung der Magnesiumkonzentration keine Wirkung auf die hormoninduzierte Freisetzung freier Fettsäuren. Das erscheint etwas überraschend, da Magnesium-Ionen für die Bildung des zyklischen 3',5'-AMP notwendig sind (13). Offenbar reichen jedoch die physiologischerweise im Gewebe vorhandenen Magnesiummengen aus, um zumindest über den Zeitraum von 3 Stdn. eine optimale Lipolyse zu ermöglichen.

In den von uns untersuchten Bereichen erfolgt bei der Spontanlipolyse die Freisetzung freier Fettsäuren proportional zur vorgegebenen Fettgewebsmenge, da es sich hier um einen allein vom Fettgewebe abhängigen Vorgang handelt.

Bei der hormoninduzierten Lipolyse dagegen lag die kritische Fettgewebsmenge bei $150 \mathrm{mg}$; von da ab war keine wesentliche Steigerung der Freisetzung freier Fettsäuren mehr möglich. Nach Hrrsch (8) muß man annehmen, daß hierbei Lipogenesevorgänge eine zunehmende Rolle spielen, da diese von der Fettgewebsmenge abhängen. Es ist bekannt, daß z. B. das lipolytisch wirkende Adrenalin gleichzeitig auch über einen stärkeren Glucose-Abbau stimulierend auf die Lipogenese aus freien Fettsäuren wirkt $(7,14)$. Die andere Möglichkeit für die im Verhältnis zu größeren Fettgewebsmengen geringere Freisetzung freier Fettsäuren - nämlich der mangelhafte Kontakt des Gewebes mit dem. lipolytisch wirkenden Hormon - scheidet auf Grund der Struktur des epididymalen Fettgewebes der Ratte aus. Albumin als Akzeptorprotein ist für die Freisetzung freier Fettsäuren aus dem Fettgewebe eine notwendige Voraussetzung (4). Nach Rudman (15) beträgt die maximale Bindungskapazität des Albumins für freie Fettsäuren 2,7 Mol/Mol Albumin. Damit sind unsere Ergebnisse erklärt, bei denen bereits nach $2 \mathrm{Stdn}$. bei einer Albuminkonzentration von $2 \mathrm{~g} / 100 \mathrm{ml}$ im Medium ein weiterer signifikanter Anstieg der freien Fettsäuren im Vergleich zu einer 5proz. Albuminkonzentration nicht mehr möglich ist; die Aufnahmekapazität des Albumins scheint zu diesem Zeitpunkt bereits erschöpft zu sein. VAUGHAN (16) nimmt als optimale Albuminkonzentration $30 \mathrm{mg} / \mathrm{ml}$ an.

'Da die Lipolyse nach den gegenwärtigen Vorstellungen durch eine Lipase - oder ein Lipasesystem - bewirkt wird, war die Temperaturempfindlichkeit dieses Systems zu prüfen. In Übereinstimmung stehen unsere Ergebnisse mit denen von Mosinger (9), der in einem Temperaturbereich von $6^{\circ}-20^{\circ}$ keine Ânderung der Spontanlipolyse, wohl aber einen Anstieg der adrenalinstimulierten Freisetzung freier Fettsäuren fand, wobei wir dieses Ver- 
halten auch bei $23^{\circ}$ feststellten. $\mathrm{Zu}$ diesen Befunden' passen gut die Beobachtungen von JoEL (17), der während der Lipolyse bei $37^{\circ}$ einen deutlich höheren Sauerstoffverbrauch feststellte als bei $8^{\circ}$. Für das experimentelle Vorgehen bei der in-vitro-Lipolyse läßt sich aus diesen Befunden ableiten, daß bei der hormoninduzierten Lipolyse eine genaue Temperatureinstellung $z$ wischen $30^{\circ}$ und $40^{\circ}$ nicht erforderlich ist. Die Feststellung von MosINGER (9), daß bei nicht maximaler Adrenalinkonzentration im Medium die Freisetzung freier Fettsäuren linear zur Zeit verläuft, trifft nach unseren Untersuchungen auch für die Spontanlipolyse und die durch ACTH stimulierte Lipolyse zu. Da jedoch im allgemeinen die Inkubation nicht länger als $3 \mathrm{Stdn}$. durchgeführt wird, gilt das auch für maximale Hormon- dosen; nur ist hierbei der wesentlich steilere Anstieg der freien Fettsäuren bereits in der ersten Std. zu berücksichtigen.

Wenn auch alle Untersuchungen am epididymalen Fettgewebe der Ratte vorgenommen wurden, ist nach den Mitteilungen aus der Literatur und unseren eigenen Ergebnissen eine Übertragung dieser Lipolysebedingungen auf andere Fettgewebsarten und andere Species möglich.

Möglicherweise läßt sich die geringere Ansprechbarkeit des menschlichen Fettgewebes auf lipolytische Hormone $(9,18)$ durch Modifikation einzelner Inkubationsbedingungen verbessern.

Herrn Dr. MrLesch, Farbwerke Hoechst, danken wir für die Überlassung von Rinderalbumin Behring.

\title{
Literatur
}

1. Gordon, R. S. und A. Cherkers, Proc. Soc. exp. Biol. Med. 97, 150 (1958). - 2. Whrte, J. E. und F. L. ENGEL, Proc. Soc. exp. Biol. Med. 99, 375 (1958). - 3. Schwandt, P., Th. Hartmann und H. J. Kari, Zschr. exper. Med. 143, 79 (1967). - 4. CAMPBell, J., A. D. Marticcr und G. R. Green, Biochem. J. 93, 183 (1964). 5. Dole, V. P. und H. Meinertz, J. biol. Chemistry 235, 2595 (1960). - 6. Rudman, D., L. A. Garcia, S. J. Brown, M. F. Malkin und W. Perl, J. Lipid Res. 5, 28 (1964). - 7. LynN, S., R. M. MACLeAd und R. H. Brown, J. biol. Chemistry 235, 1904 (1960). - 8. Hirsch, J., in: Handbook of Physiology, Section 5, 455 (1965), Williams \& Wilkins Company, Baltimore, Maryland, USA. - 9. Mosinger, B., in: Handbook of Physiology, Section 5 ,
601 (1965). - 10. Christophe, J.: Contribution à la biochemie des obésités experimentales, Editions Arscia S. A., Bruxelles, 1961. 11. Lopez, E., J. E. White und F. L. ENGeL, J. biol. Chemistry 234, 2254 (1959). - 12. Rodbel, M., in: Handbook of Physiology, Section 5, 471 (1965). - 13. Sutherland, E. W. und G. A. RoBison, Pharmacol. Rev. 18, 145 (1966). - 14. WINEGRAD, A. J., Vitamins and Horm., N. Y. 20, 141 (1962). - 15. Rudman, D., J. Lipid Res. 4, 119 (1963). - 16. VaughaN, M., in: Handbook of Physiology, Section 5, 239 (1965). - 17. JoEL, C. D., in: Handbook of Physiology, Section 5, 59 (1965). - 18. Schwandt, P. und Th. HartmanN, unveröffentlichte Versuche.

\section{Vorkommen von UDP-Glucuronyltransferasen in der Magenschleimhaut des Menschen}

\author{
Von W. HofFManN und H. Breuer \\ Aus der Abteilung für Klinische Chemie und Biochemie der Cbirurgischen Universitätsklinik und Poliklinik Bonn \\ (Direktor: Prof. Dr. A. Gütgemann)
}

(Eingegangen am 24. August 1967)

Nach Inkubation von $\left[4^{14} \mathrm{C}\right] 17 \beta$-Östradiol mit der Cytoplasma-Fraktion (105000 $\mathrm{g}$-Uberstand) der pylorusnahen Magenschleimhaut des Menschen in Gegenwart von Uridin-5'-diphospho-glucuronat konnte $\left[4-{ }^{14} \mathrm{C}\right] 17 \beta$-Östradiol-17 $\beta$-monoglucuronid nachgewiesen werden; $\left[4^{14} \mathrm{C}\right] \mathrm{O}$ striol ergab unter den gleichen Bedingungen $\left[4{ }^{14} \mathrm{C}\right]$ Östriol-17 $\beta$-monoglucuronid. In Versuchen mit der MikrosomenFraktion der Magenschleimhaut entstanden ebenfalls $\left[{ }^{14} \mathrm{C}\right] 17 \beta$-Östradiol-17 $\beta$-monoglucuronid und $\left[4-{ }^{14} \mathrm{C}\right]$ Östriol-17 $\beta$-monoglucuronid; außerdem wurde $\left[4{ }^{14} \mathrm{C}\right]$ Östriol-16 $\alpha$-monoglücuronid gebildet. $\left[4{ }^{14} \mathrm{C}\right]$ Ostron wurde weder durch die Cytoplasma- noch durch die Mikrosomen-Fraktion glucuronidiert. Die Versuche zeigen, daß in der Magenschleimhaut des Menschen cytoplasmatische (lösliche) und mikrosomale UDP-Glucuronyltransferasen vorhanden sind. Die Glucuronyltransferasen besitzen eine ausgeprägte Gruppenspezifität und greifen nur alkoholische Hydroxylgruppen phenolischer Steroide an. Demnach bestehen zwischen den Glucuronyltransferasen der Magenschleimhaut und der Dünndarmschleimhaut des Menschen Unterschiede.

The enzymic formation of $\left[4{ }^{14} \mathrm{C}\right] 17 \beta$-oestradiol $17 \beta$-monoglucuronide was demonstrated after incubation of $\left[4{ }^{14} \mathrm{C}\right] 17 \beta$-oestradiol with the cytoplasmic fraction ( $105000 \mathrm{~g}$ supernatant) of human gastric mucosa in the presence of uridine diphosphate glucuronic acid. Under similar conditions, $\left[4^{14} \mathrm{C}\right]$ oestriol yielded $\left[4^{14} \mathrm{C}\right]$ oestriol $17 \beta$-monoglucuronide. Similarly, in experiments with the microsomal fraction, $[4-14 \mathrm{C}] 17 \beta$-oestradiol $17 \beta$-monoglucuronide and $\left[4{ }^{14} \mathrm{C}\right]$ oestriol $17 \beta$-monoglucuronide were formed from $\left[4{ }^{14} \mathrm{C}\right] 17 \beta$-oestradiol and $\left[4{ }^{14} \mathrm{C}\right]$ oestriol, respectively; in addition, $\left[{ }^{14} \mathrm{C}\right]$ oestriol $16 \alpha$-monoglucuronide was detected. No glucuronidation of $[4-14 \mathrm{C}]$ oestrone occurred in either the cytoplasmic or the microsomal fractions. These experiments demonstrate that the human gastric mucosa contains soluble as well as microsomal UDP-glucuronyltransferases. The glucuronyltransferases possess a pronounced substrate specifity and attack only alcoholic hydroxyl groups of phenolic steroids. The results reported here show that differences exist between the glucuronyltransferases of the gastric mucosa and the intestinal mucosa of man. 